\title{
TÉCNICAS DO LEAN THINKING APLICADAS AOS PROCESSOS LOGÍSTICOS DE UM TRANSPLANTE DE FÍGADO
}

\author{
Lean Thinking Techniques Applied to the Logistics Processes of a Liver Transplantation \\ Vera Lucia Monteiro, ${ }^{1,2}$ Helder José Lessa Zambelli³, Ilka de Fátima Santana Ferreira Boin ${ }^{3}$, Orlando Fontes Lima Junior²
}

\begin{abstract}
RESUMO
Objetivos: Os objetivos desta pesquisa foram mapear um transplante de fígado, fazer análises críticas nos aspectos logísticos envolvidos, sob a ótica da filosofia Lean e sugerir melhorias que levem à minimização de desperdícios e demais ineficiências. Métodos: A pesquisa foi realizada junto à Organização de Procura de Órgãos do Hospital das Clínicas da Universidade Estadual de Campinas. Foram acompanhados processos desde a captação do órgão até o transplante no receptor. Após o mapeamento dos casos isolados, foram feitas análises críticas e um redesenho de processo foi proposto, visando reduzir o seu lead time. Resultados: Um mapa hipotético de valor futuro foi elaborado, representando processos com menos desperdícios. Finalmente, foram comparados os lead times inicial e final e algumas considerações foram feitas. Conclusão: Espera-se com este estudo validar a aplicabilidade das técnicas do lean thinking como capazes de produzir melhorias nos processos da logística de transplantes, assim como vem acontecendo em outras atividades da área da saúde.
\end{abstract}

Descritores: Logística, Métodos, Transplante de Fígado, Avaliação em Saúde.

\footnotetext{
Instituições:

1 Faculdade de Tecnologia de São José dos Campos (FATEC), São Paulo - Brasil.

${ }^{2}$ Laboratório de Aprendizagem em Logística e Transportes (LALT), Faculdade de Engenharia Civil, Arquitetura e Urbanismo, Departamento de Geotecnia e Transportes, Universidade Estadual de Campinas (UNICAMP), São Paulo - Brasil.

${ }^{3}$ Faculdade de Ciências Médicas, Universidade Estadual de Campinas (UNICAMP), São Paulo - Brasil.
}

\section{Correspondência:}

Vera Lucia Monteiro

Rua Albert Einstein, 951, Sala 08, Caixa Postal 6021

Cidade Universitária Zeferino Vaz - CEP 13083-852 - Barão Geraldo - Campinas/SP

Telefax: (19) 3521-2346

E-mail: monteiro_vera@uol.com.br

Recebido em: 29.12.2009

Aceito em: 15.01.2010

\section{INTRODUÇÃO}

Demonstrar a aplicabilidade das técnicas do lean thinking no processo de transplante de órgãos, visando eliminar desperdícios e redução de tempos de ciclo foi o alvo deste trabalho.

Neste estudo foram analisados os processos logísticos envolvidos nas fases de um transplante de fígado, sob a ótica da filosofia Lean. Essas análises buscaram identificar o que é valor em cada etapa e propor melhorias no processo.

O Sistema Nacional de Transplantes (SNT) tem manifestado publicamente seu compromisso de melhoria contínua, não somente no que diz respeito às técnicas médicas, mas também no que se relaciona ao aperfeiçoamento dos processos logísticos. ${ }^{1}$

No Brasil, a lista de espera por transplantes tem crescido ano a ano e, segundo o SNT, a segunda maior causa de não efetivação das doações de órgãos são problemas relacionados à logística, conforme demonstra a Tabela 1. 
Tabela 1. Causas da não-efetivação dos transplantes depois do diagnóstico de morte encefálica.

\begin{tabular}{|l|c|c|c|c|c|c|}
\cline { 2 - 7 } \multicolumn{1}{c|}{} & \multicolumn{2}{c|}{2002} & \multicolumn{2}{c|}{$\mathbf{2 0 0 3}$} & \multicolumn{2}{c|}{2004} \\
\cline { 2 - 7 } \multicolumn{1}{c|}{} & $\mathbf{N}^{\mathbf{0}}$ & $\mathbf{\%}$ & $\mathbf{N}^{\mathbf{0}}$ & $\mathbf{\%}$ & $\mathbf{N}^{\mathbf{0}}$ & $\mathbf{\%}$ \\
\hline $\begin{array}{l}\text { Total de Notificações de } \\
\text { Morte Encefálica }\end{array}$ & 4.346 & $100 \%$ & 4.771 & $100 \%$ & 5.050 & $100 \%$ \\
\hline $\begin{array}{l}\text { Doações Não Efetivadas - } \\
\text { Negativa Familiar }\end{array}$ & 1.387 & $32 \%$ & 1.504 & $32 \%$ & 1.282 & $25 \%$ \\
\hline $\begin{array}{l}\text { Doações Não Efetivadas - } \\
\text { Aspectos Logísticos }\end{array}$ & 1.159 & $27 \%$ & 1.129 & $24 \%$ & 1.208 & $24 \%$ \\
\hline $\begin{array}{l}\text { Doações Não } \\
\text { Efetivadas - Parada } \\
\text { Cardiorrespiratória }\end{array}$ & $\mathbf{6 5 3}$ & $\mathbf{1 5 \%}$ & $\mathbf{7 7 9}$ & $\mathbf{1 6 \%}$ & $\mathbf{8 4 4}$ & $\mathbf{1 7 \%}$ \\
\hline $\begin{array}{l}\text { Doações Não Efetivadas - } \\
\text { Sorologia }\end{array}$ & 188 & $4 \%$ & 171 & $4 \%$ & 298 & $6 \%$ \\
\hline $\begin{array}{l}\text { Doadores Efetivos de } \\
\text { Órgãos }\end{array}$ & 959 & $22 \%$ & 1.198 & $25 \%$ & 1.417 & $28 \%$ \\
\hline
\end{tabular}

Fonte: Adaptado de Sistema Nacional de Transplantes (SNT), 2005.

Finalmente, longe de tratar de aspectos técnicos da área médica, o estudo ressaltou a complementaridade das diversas áreas do conhecimento, quando se busca a melhoria dos resultados em um processo.

\section{LEAN THINKING E A LOGÍSTICA}

O termo "enxuto" como tradução de lean surgiu na história dos negócios para qualificar o sistema Toyota de fabricação. Tal sistema era lean por uma série de razões: exigia menos esforço humano para projetar e produzir os veículos, reduzia os investimentos por unidade de produção, trabalhava com menos fornecedores, operava com menos peças em estoque em cada etapa do processo produtivo, diminuía os defeitos. O número de acidentes de trabalho também era menor e demonstrava significativas reduções de tempo entre a concepção do produto e seu lançamento em escala comercial, entre o pedido feito pelo cliente e a entrega e entre a identificação de problemas e a resolução dos mesmos. ${ }^{2}$

O termo lean thinking refere-se ao "antídoto para o desperdício". ${ }^{3}$ $\mathrm{O}$ desperdício refere-se a qualquer atividade humana que não acrescenta valor. O conceito de desperdício deve ser ampliado, passando a incluir não apenas atividades humanas, como também qualquer outro tipo de atividade e recursos usados indevidamente e que contribuem para o aumento de custos, de tempo e da não satisfação do cliente.

Como a repercussão econômica mais visível da adoção do conceito lean sempre foi a diminuição de estoques, através de entregas mais freqüentes e redução dos lotes de compra e/ou de fabricação, surgiu no campo da logística o conceito do "ressuprimento enxuto", ou "logística enxuta". O conceito de logística enxuta é mais amplo e envolve iniciativas que visam à criação de valor para os clientes mediante um serviço logístico realizado com menor custo total para os integrantes da cadeia de suprimentos. ${ }^{2}$

A Logística Lean atua sobre os excessos de estoques, movimentações desnecessárias, tempos não-planejados de ociosidade e de troca de turnos, que também são todos sintomas de desperdício. ${ }^{4}$ Algumas das ferramentas típicas utilizadas na implementação da filosofia lean na área as saúde são apresentadas no quadro 1.

Quadro 1. Ferramentas utilizadas no Lean Thinking aplicado à área da saúde

\begin{tabular}{|c|c|}
\hline \multicolumn{2}{|c|}{ Ferramentas do Lean Healthcare } \\
\hline $5 \mathrm{~S}$ & Sistema Arquivo de Papel \\
\hline Fluxo Contínuo & Leiaute Físico \\
\hline Tempo de Ciclo & Saída Previsível \\
\hline Verificação de Erros & Solução de Problema \\
\hline Metas e Resultados & Sistemas Puxados \\
\hline Cartão de Meta & Seis Sigma \\
\hline Interrupções e Chegadas Aleatórias & Padronização de Trabalho \\
\hline Just in time & Tempo Takt \\
\hline Eventos Kaizen & Mapa de Valor \\
\hline Kanban para Suprimentos & Controle Visual \\
\hline Nivelamento & Desperdício \\
\hline Técnicas de Medição & Balanceamento de Carga de \\
\hline
\end{tabular}

\section{Mapeamento do Fluxo de Valor}

As corporações industriais e as organizações em geral têm despendido consideráveis esforços e recursos no sentido de promover melhoria contínua de seus processos, e assim garantir sua competitividade e uma posição mais sólida no mercado. Uma ferramenta introduzida por Mike Rother e John Shook em 1999, chamada de Mapeamento de Fluxo de Valor (Value Stream Mapping) tem papel fundamental no processo de construção do cenário atual e perspectiva de um cenário futuro, com menos desperdícios para as organizações.

Conceitua-se o mapeamento de fluxo de valor ou value stream mapping como uma ferramenta de identificação das atividades específicas que ocorrem ao longo do fluxo de valor referente a um processo.

O objetivo principal da ferramenta de Mapeamento do fluxo de valor é avaliar cada etapa dos processos como sendo criadora ou não de valor e demonstrar quais são os pontos que podem ser alterados, para que o processo seja otimizado. ${ }^{5}$

No Lean Healthcare Pocket Guide XL, valor é a percepção do paciente (cliente) sobre o grau de atendimento de suas necessidades, ou seja, as atividades agregadoras de valor que satisfazem os pacientes através do completo atendimento de suas necessidades. ${ }^{6}$

\section{Lean na área da saúde e os transplantes de órgãos}

A filosofia Lean, outrora aplicada apenas aos setores produtivos, pode ser perfeitamente estendida a todos os setores e todas as organizações, inclusive àquelas estritamente prestadoras de serviços, como a área da saúde, pois a aplicação de seus conceitos resulta em agilidade, sensibilidade aos desejos dos clientes e, portanto, maior potencial competitivo. ${ }^{7}$ 
Os conceitos ligados à filosofia Lean possuem forte potencial para representar um papel importante na melhoria de desempenho das atividades na área de saúde.

A Lean Logistics identifica pontos geradores de desperdício e ajuda a cumprir objetivos primordiais da logística, que são atendimento com a maior rapidez possível e com qualidade, sem, no entanto, incorrer em gastos exagerados e/ou desnecessários, otimizando suas tarefas e padronizando procedimentos. ${ }^{8}$

A Lean Enterprise Academy é uma entidade sem fins lucrativos que tem por objetivo difundir os conceitos lean para todos os tipos de organização. Essa entidade organiza anualmente o Lean Healthcare Forum, evento mundial onde são divulgados trabalhos na área da saúde desenvolvidos com o uso das técnicas Lean.

Os resultados divulgados nesse fórum são muitos e se referem a várias atividades de sistemas hospitalares, da triagem de pacientes na emergência do Flinders Medical Center (Austrália) até o sistema de coleta de material para exames clínicos e distribuição de resultados no Intermountain Health Care System (EUA), todos apresentando ganhos comprovados, não somente em termos de simplificação e agilidade de seus processos e consequente aumento na satisfação dos clientes, mas também com importantes reduções de custo.

Do ponto de vista logístico, o transplante envolve processo de acondicionamento, armazenagem e transporte, levando em consideração o tempo de isquemia (tempo em que o órgão se mantém viável para transplante a partir da interrupção da circulação sanguínea) de cada órgão e as distâncias entre doadores e receptores. Envolve também o provimento de recursos específicos, como agendamento de salas cirúrgicas, materiais, equipamentos e pessoal especializado e também todo fluxo de informações entre os envolvidos. ${ }^{9}$

$\mathrm{Na}$ logística dos transplantes de órgãos foram identificados cinco processos-chave para estudo e mapeamento do fluxo de valor. Esses processos-chave têm características de componentes do processo de transplante e, portanto, são independentes e ao mesmo tempo interligados, de forma que o desempenho de cada um influencia o resultado final do todo. ${ }^{10}$

Esses processos são: captação do órgão doado, acondicionamento, transporte, preparação e transplante no receptor, além da troca de informação entre equipes.

O propósito do Lean é eliminar de um processo todos os desperdícios ou atividades que não agregam valor ao cliente. Os desperdícios na área da saúde estão distribuídos em oito categorias, conforme cita o Lean Healthcare Pocket Guide XL: ${ }^{6}$

a. Serviços desnecessários ou superprodução

b. Erros ou Defeitos

c. Atrasos ou Esperas

d. Movimentações Desnecessárias

e. Excesso de processamento

f. Excesso de Estoque

g. Transporte em excesso

h. Criatividade não utilizada

Esses desperdícios foram procurados nos processos mapeados, com o objetivo de serem minimizados através da implementação das melhorias sugeridas.

\section{SITUAÇÃO DOS TRANSPLANTES NO BRASIL}

Conforme os dados da Associação Brasileira de Transplante de Órgãos (ABTO), o Brasil é atualmente o terceiro no mundo em número de transplantes, antecedido pelos Estados Unidos e China. ${ }^{11}$

De acordo com o SNT, o Brasil vem apresentando crescimento no setor de transplantes. Esse crescimento é conseqüência da conscientização da população brasileira, da atuação competente de equipes e instituições autorizadas pelo Sistema Nacional de Transplantes do Ministério da Saúde e da regulação do SNT, fundamentada na legislação vigente.

Tabela 2. Evolução anual dos transplantes (em número absoluto).

\begin{tabular}{|c|c|c|c|c|c|c|c|c|c|}
\hline Ano / Órgão & $\mathbf{2 0 0 0}$ & $\mathbf{2 0 0 1}$ & $\mathbf{2 0 0 2}$ & $\mathbf{2 0 0 3}$ & $\mathbf{2 0 0 4}$ & $\mathbf{2 0 0 5}$ & $\mathbf{2 0 0 6}$ & $\mathbf{2 0 0 7}$ & $\mathbf{2 0 0 8}$ \\
\hline Coração & 121 & 131 & 150 & 175 & 202 & 180 & 148 & 159 & 200 \\
\hline Fígado & 486 & 564 & 676 & 816 & 965 & 952 & 1036 & 1014 & 1175 \\
\hline Pâncreas & 46 & 119 & 193 & 217 & 251 & 180 & 181 & 162 & 166 \\
\hline Pulmão & 25 & 23 & 34 & 42 & 46 & 45 & 49 & 46 & 53 \\
\hline Rim & 2912 & 3117 & 3042 & 3185 & 3487 & 3372 & 3287 & 3457 & 3808 \\
\hline Total & 3590 & 3954 & 4095 & 4435 & 4951 & 4729 & 4701 & 4838 & 5402 \\
\hline Aumento (\%) & 10,14 & 3,57 & 8,30 & 11,63 & $-4,48$ & $-0,59$ & 2,91 & 11,66 \\
\hline \multicolumn{10}{|c|}{ Aumento (\%) de 2000 a 2008 = 50,47 } \\
\hline
\end{tabular}

O Sistema Nacional de Transplante (SNT) adotado no Brasil tem a concepção de fila única, com o propósito de garantir o acesso universal, justo e gratuito aos órgãos disponíveis. Assim, ao administrar as questões legais, morais e técnicas que envolvem todo o processo de transplante, o SNT visa garantir que os órgãos doados cheguem em tempo hábil a receptores previamente listados em conformidade com as determinações legais.

Ao SNT cabe o papel de gerenciar a lista única nacional de receptores, com todas as indicações necessárias à busca de órgãos; assim como é de sua responsabilidade conceder a autorização aos estabelecimentos de saúde e equipes especializadas para realizar a captação, transporte e transplante do órgão. Todo esse processo inicia-se após identificação da morte encefálica do potencial doador e sua notificação à Central de Transplante. E sua efetividade depende da rapidez e precisão com que o processo é conduzido.

Este estudo teve por premissa básica a afirmação de que para transplantes de órgãos, tempo é a principal e mais importante restrição. Desde o momento em que o processo de doação é iniciado, todas as atividades da captação ao transplante devem ser conduzidas dentro do mais curto período de tempo. ${ }^{12}$

Com esta pesquisa, espera-se comprovar que mesmo em ambientes não controlados, como é o caso do processo de transplantes de órgãos, as técnicas do lean thinking são capazes de produzir melhorias como a minimização dos desperdícios, agilização dos processos logísticos e, por conseqüência, a redução dos tempos de ciclo. 


\section{MÉTODOS}

As principais atividades basearam-se nas coletas de dados (primários e secundários). Os dados primários foram obtidos por meio de observação direta, pelo acompanhamento do caso escolhido (processos de captação ao transplante do órgão) e de entrevistas com os profissionais de saúde envolvidos. Os dados secundários foram obtidos em publicações especializadas dos setores de lean e da saúde, e em sites que abordam o tema transplantes, especialmente o site da ABTO.

Conforme a figura 1 demonstra, após os processos isolados (captação, acondicionamento, transporte, preparação e transplante) terem sido acompanhados, foi elaborado um mapa de fluxo de valor atual hipotético. Então, análises críticas foram feitas, e em seguida, foi sugerida aplicação de ferramentas típicas do lean, visando reduzir alguns dos desperdícios identificados.

Figura 1. Fluxograma do Estudo de Caso.

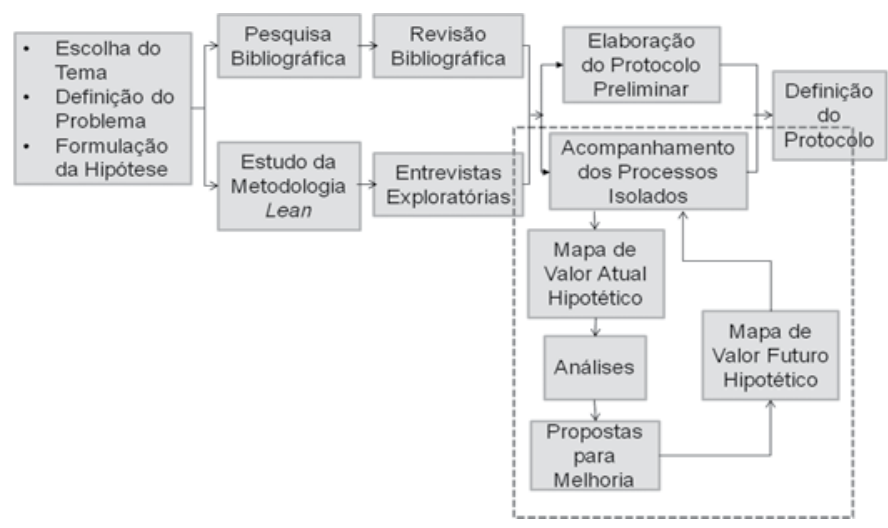

Após isso, foi proposto um redesenho do processo, representado por um mapa de valor futuro hipotético, o qual deverá ser aplicado a outro caso, para comprovação dos ganhos apontados.

A idéia da filosofia lean é repetir essas etapas várias vezes, sempre procurando detectar e eliminar novos desperdícios, num processo de obtenção de melhoria contínua (Kaizen).

\section{RESULTADOS}

Conforme já foi citado anteriormente, foram acompanhados os processos de captação, acondicionamento, transporte, preparação e transplante de fígado. A seguir, estão as análises críticas feitas a cada processo observado e a descrição dos desperdícios observados.

\section{Processo de Captação}

Durante a captação foi detectado que alguns itens não estavam disponíveis no momento da perfusão do órgão e tiveram que ser solicitados pelo médico, atrasando a operação. Também foi observada falta de alguns materiais de apoio ao ambiente cirúrgico. Esse fato ocasionou excessos de movimentação, inclusive atrapalhando deslocamentos dos profissionais médicos dentro do centro cirúrgico. Esses fatos denotam falta ou falha no treinamento do pessoal de apoio médico.

\section{Processo de Acondicionamento}

Durante o processo de embalagem foi detectado desconhecimento ou descumprimento de procedimentos. Trabalhos desnecessários foram executados por não haver embalagens adequadas aos diferentes tamanhos de órgãos e por não haver uma forma de fechamento da embalagem que agilize esse processo.

\section{Processo de Transporte}

A etapa de transporte é realizada hoje com um veículo comum, de uma empresa contratada para realização desse processo. Por isso, o transporte está sujeito a problemas de trânsito e congestionamentos diversos ao longo dos trajetos.

Como exemplo, o transporte do fígado acompanhado saiu de uma cidade a cerca de $200 \mathrm{~km}$ de distância do hospital onde ocorreria o transplante e levou 3 horas e 20 minutos de viagem. O normal seria que a viagem levasse 2 horas, no máximo. $O$ fato de ter ocorrido um congestionamento por excesso de veículos na estrada fez com que o processo tivesse um atraso de 1 hora e 20 minutos.

\section{Processo de Preparação}

Houve demora em avisar o médico que o órgão já estava disponível para preparação, apontando problemas de comunicação entre as equipes do hospital que recebem o órgão e a equipe médica. Devido a isso, houve espera para que o procedimento fosse iniciado.

\section{Processo de Transplante}

No processo de transplante do órgão observou-se excesso de movimentação dentro do centro cirúrgico, devido à falta de material, que não estava conforme as especificações exigidas pelo médico. Também ocorreram esperas devido à falta de produto necessário à continuidade de atividades em andamento (materiais de consumo diversos).

A falta de uma metodologia mais eficiente para entrega de soro morno ao médico gerou muita perda de material e ainda mais atrasos, pela necessidade de retrabalhos constantes durante esse processo.

\section{Processo de Troca de Informação entre Equipes}

Conforme relatado em entrevistas feitas com a equipe da Organização de Procura de Órgãos (OPO) do Hospital das Clínicas (HC) da UNICAMP, muito tempo é desperdiçado com retrabalhos e espera na troca de informação entre o hospital notificador do potencial doador e equipes da OPO. Isso acontece, principalmente, por deficiência no sistema de comunicação atual, feito por intermédio de fax e telefone.

Também não há um relatório padrão contendo todas as informações necessárias ao início do processo de doação, evitando a necessidade de contatos freqüentes para a complementação de dados.

Devido aos motivos acima, as perdas durante as trocas de informação são sempre da ordem de horas, conforme informação cedida pela OPO. Diante das descrições dos processos de transplante de órgãos e da metodologia de mapeamento do fluxo de valor, a figura 2 representa a situação atual de todo o processo e os fluxos de informações e de material. 


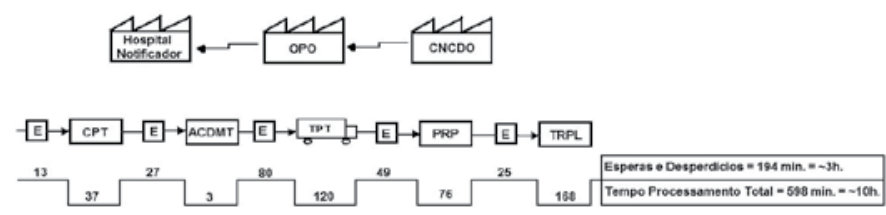

Tempo de Valor Agregado $=404 \mathrm{~min} .=\sim 7 \mathrm{~h}$. Tempo de Processamento Total corresponde ao Lead Time. CNCDO: Central de Notificação, Captação e Distribuição de Órgãos. OPO: Organização de Procura de Órgãos. E: Espera. CPT: Captação. ACDMT: Acondicionamento. TPT: Transporte. PRP: Preparação. TRPL: Transplante.

\section{DISCUSSÃO}

As propostas de melhorias listadas abaixo, seguidas das respectivas ferramentas Lean mais adequadas na provisão de melhorias, foram baseadas na análise do Mapa de Valor Atual (figura 2), desenhado através da observação direta dos processos isolados acima descritos:

a. Revisar protocolo de preparação do centro cirúrgico - 5S;

b. Preparar um check-list para ser utilizado antes das cirurgias - 5S;

c. Estabelecer rotina de envio de informação ao cirurgião responsável pelo transplante quando o órgão for entregue ao hospital, se a captação for feita por equipe diferente da equipe transplantadora - Controle Visual (CV);

d. Padronizar embalagens primárias e secundárias, de acordo com o tamanho de cada órgão e as especificações da Agência Nacional de Vigilância Sanitária (ANVISA) - Padronização (P);

e. Desenvolver uma forma de fechamento da embalagem primária e secundária, de forma a agilizar esse processo - Padronização (P);

f. Padronizar embalagem terciária (caixa isotérmica de material rígido e resistente) conforme especificações da ANVISA, com termômetro acoplado e lacre que impeça sua abertura fora do hospital, garantindo a manutenção da temperatura pelo tempo necessário ao processo de transporte - Padronização (P);

g. Criar um relatório padrão que acompanhe os documentos durante o processo de transporte, para informar a ocorrência de não conformidades que tragam riscos à conservação e integridade dos órgãos humanos ou riscos de contaminação do material e do pessoal envolvido no transporte - Padronização $(\mathrm{P})$;

h. Estabelecer instrução normativa definindo que o veículo de transporte de órgãos humanos para fins de transplante deverá ser de tipo especial devidamente equipado, de forma a prevenir danos ao órgão, seja por choques mecânicos e/ou por deslizamentos durante o percurso, além de garantir a agilidade do transporte -Padronização $(\mathrm{P})$;

i. Desenvolver um container térmico para soro fisiológico estéril com capacidade suficiente para atender ao procedimento de aquecimento do órgão e dessa forma melhorar e agilizar a sistemática atual de abastecimento - Fluxo Contínuo (FC); j. Implantar um sistema de troca eletrônica de dados e documentos (EDI - Eletronic Data Interchange) via web, de forma a garantir agilidade, segurança, acuracidade e economia às trocas de informação entre as equipes envolvidas - Just in Time (JIT) /EDI;

A implementação das sugestões acima visa eliminar do processo os desperdícios detectados. Um redesenho de processo com menos desperdícios é representado através de seu mapa de valor futuro, conforme demonstrado na figura 3.

Figura 3. Mapa do Fluxo de Valor Futuro

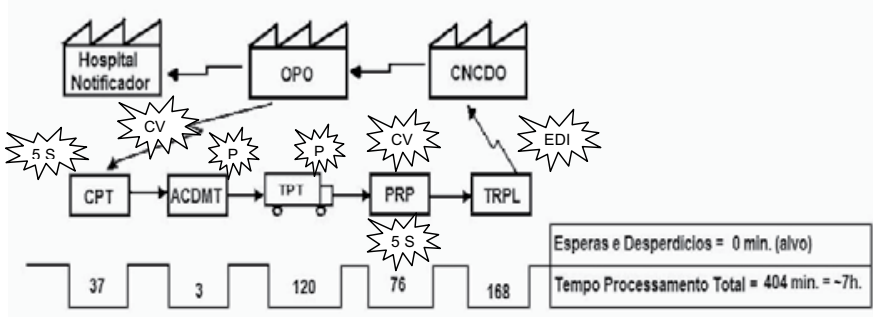

Tempo de Valor Agregado $=404 \mathrm{~min} .=\sim 7 \mathrm{~h}$. Tempo de Processamento Total corresponde ao Lead Time. CNCDO: Central de Notificação, Captação e Distribuição de Órgãos.OPO: Organização de Procura de Órgãos. E: Espera. CPT: Captação. ACDMT: Acondicionamento. TPT: Transporte. PRP: Preparação. TRPL: Transplante. EDI: Eletronic Data Interchang. CV: Controle Visual. P: Padronização.

\section{CONCLUSÃO}

Baseado nos casos isolados acompanhados e nas entrevistas realizadas, conclui-se que mediante a implementação das sugestões descritas é possível a obtenção de um ganho em Tempo de Ciclo Total superior a $30 \%$, ou seja, de três horas.

Esse ganho em ciclo deverá ser comprovado através do acompanhamento de outros processos, onde o mapa de valor atual deverá ser o mapa de valor futuro proposto neste estudo. A Metodologia Lean orienta a continuar mapeando os processos, detectando e eliminando outros desperdícios, de forma a implementar uma cultura de melhoria contínua entre os grupos e suas respectivas atividades (Kaizen).

As sugestões apresentadas neste estudo referem-se exclusivamente às atividades de Logística. Outros estudos poderão comprovar a eficácia do método, em se tratando de técnicas e procedimentos médicos. 


\section{ABSTRACT}

Purpose: The purpose of this research was to make a map of a liver transplantation process, aiming to make a critical analysis on the involved logistics issues, always under the focus of the Lean philosophy, and to suggest improvements that could lead to minimize the waste and other inefficiencies. Methods: The research was developed in partnership with the Hospital das Clínicas Organ Procurement Organization of the State University in Campinas. Procedures have been followed from the organ procurement up to the transplantation in the recipient. After making a map of isolated cases, critical analyses were made, and a process redesign was proposed in order to reduce the lead time. Results: This redesign was represented by a hypothetical current state value stream mapping. Finally, we compared the initial lead time to the final lead time, and some conclusions have been taken. Conclusions: We hope this study will validate the applicability of lean thinking techniques as able to produce improvements in the logistics processes of transplantation proceedings, as it is happening in other healthcare activities.

Keywords: Logistic, Methods, Liver Transplantation, Health Evaluation.

\section{REFERÊNCIAS}

1. Sistema Nacional de Transplantes [homepage na Internet]. São Paulo; [acesso em 2009 Jun 16] Disponível em: http://www.dtr2001.saude.gov.br/transplantes

2. Figueiredo K. A Logística Enxuta. Centro de Estudos em Logística - COPPEAD/ UFRJ 2006. [acesso em 2009 May 15] Disponível em: http://www.centrodelogistica. com.br

3. Womack JP., Jones DT. Lean thinking. Simon \& Schuster: UK; 2003.

4. Takeuchi NE. Lean Institute [homepage na Internet]. Logística Lean para a Eliminação do Warehouse. 2007 [acesso em 2009 May 30] Disponível em: http:// www.lean.org.br

5. Rother M, Shook J. Aprendendo a Enxergar - Mapeando o fluxo de valor para agregar valor e eliminar o desperdício. São Paulo: Lean Institute; 1999.

6. Redfild D, Holmes S. Lean Healthcare Pocket Guide XL - Tools for the Elimination of Waste in Hospitals, Clinics and Other Healthcare Facilities. USA: ELHI; 2004.
7. Dickson EW, Singh S, Cheung DS, Wyatt CC, Nugent AS. Application of Lean Manufacturing Techniques in the Emergency Department. The Journal of Emergency Medicine. 2009;37:177-82.

8. Silberstein ACL. Princípios Enxutos em Serviços de Saúde no Brasil [dissertação de Mestrado]. Rio de Janeiro: Instituto COPPEAD de Administração da Universidade Federal do Rio de Janeiro; 2006

9. Ratz W. Indicadores de Desempenho na Logística do Sistema Nacional de Transplantes: Um estudo de caso [dissertação de Mestrado]. São Paulo: Universidade Federal de São Carlos; 2006.

10. Meredith DD et al. Design and Planning of Engineering Systems. New Jersey: Prentice Hall; 1985

11. Associação Brasileira de Transplantes de Órgãos (ABTO) [homepage na Internet]. São Paulo. [acesso em 2009 Oct 22). Disponível em: http://www.abto.org.br

12. Fuzzati R. Organ Transplantation Management. Swiss Federal Institute of Technology Lausanne (EPFL). Technical Report. 2005; No: IC/2005/022. 